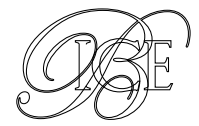

\section{SALDO FINANCIERO ESPAÑA-UNIÓN EUROPEA EN EL AÑO 2019}

El artículo que se presenta a continuación realiza una descripción de los principales flujos de ingresos y pagos que se han producido durante el año 2019 entre España y la Unión Europea, analizándose los distintos factores explicativos de su evolución y el resultado final del saldo financiero alcanzado por España en dicho año. También se mencionan los principales acontecimientos que han afectado al presupuesto de la Unión Europea del ejercicio en cuestión y sus implicaciones para el saldo financiero con España.

Palabras clave: Presupuesto General de la Unión Europea, flujos financieros, saldo financiero, Presupuestos Generales del Estado, fondos estructurales, recursos propios, IVA, RNB, marco financiero plurianual.

Clasificación JEL: E50, H61.

\section{Introducción}

España, como Estado miembro de la Unión Europea, al igual que el resto de los países que la componen, participa, por un lado, en la financiación de la Unión Europea en la medida que le corresponde $y$, por otro, recibe fondos de aquella en el marco del desarrollo de las políticas comunes.

El Presupuesto de la Unión Europea se financia básicamente con los denominados recursos propios comunitarios, que son aportados por todos los Estados miembros y que están constituidos por los recursos propios

\footnotetext{
* Este artículo ha sido elaborado por Aurora Gallego Sánchez, Cuerpo de Diplomados Comerciales del Estado.

Versión de abril de 2020.

DOI: https://doi.org/10.32796/bice.2020.3123.7000
}

tradicionales (derechos de aduana y cotizaciones sobre el azúcar e isoglucosa), el recurso IVA y el recurso RNB (renta nacional bruta). A su vez, la Unión Europea realiza una serie de gastos en los Estados miembros con cargo a sus diferentes líneas de actuación.

La mayor parte de estos flujos financieros tienen su reflejo tanto en los Presupuestos Generales del Estado español (PGE) como en el Presupuesto General de la Unión Europea (PGUE), aunque algunos escapan a esta doble consignación. Es el caso, por ejemplo, de la contribución anual que España realiza al Fondo Europeo de Desarrollo (FED), que no tiene reflejo en el Presupuesto de la Unión Europea aunque sí en el Presupuesto del Estado español; asimismo, los fondos estructurales para acciones cofinanciadas por comunidades autónomas, corporaciones locales o empresas $\triangle$ 
se transfieren directamente a estos entes a través de la Dirección General del Tesoro y quedan sin reflejo en los Presupuestos Generales del Estado.

\section{El marco financiero del Presupuesto de la Unión Europea para el año 2019}

El presupuesto de 2019 es el penúltimo que se regirá por el marco financiero plurianual 2014-2020, aprobado por el Reglamento 1311/2013 y modificado por el Reglamento del Consejo $n$. 2015/623, de 21 de abril de 2015 (publicado en el Diario Oficial de la Unión Europea L103 con fecha 22 de abril de 2015). Junto con este Reglamento, se aprobó el Acuerdo Interinstitucional de 2 de diciembre de 2013, sobre disciplina presupuestaria y buena gestión financiera, que incluye medidas dirigidas a mejorar el desarrollo del procedimiento presupuestario anual y complementa el Reglamento del Marco Financiero Plurianual (MFP).

El Marco Financiero Plurianual define, para cada periodo de programación, los límites máximos anuales para los créditos de compromiso de cada rúbrica o categoría de gasto, así como el techo para el total de créditos de pago en cada ejercicio presupuestario; y en el procedimiento presupuestario anual se determina el importe exacto de los gastos y su distribución entre las líneas presupuestarias para el año de que se trate.

Estas grandes orientaciones presupuestarias anuales garantizan una evolución ordenada del gasto de la Unión y son acordadas por el Parlamento Europeo y el Consejo.

El Reglamento del MFP 2014-2020 introduce diversas cláusulas relativas a la flexibilidad, concebidas para que la Unión pueda responder a determinadas situaciones imprevistas o excepcionales y permitir financiar actuaciones que no tengan cabida dentro de los límites del MFP. Los instrumentos especiales al margen de los techos de gastos acordados en el MFP 2014-2020 son:

- Reserva para Ayudas de Emergencia.

- Fondo Europeo de Adaptación a la Globalización.

- Fondo de Solidaridad de la Unión Europea.

- Instrumento de Flexibilidad.

- Margen para Imprevistos.

- Margen Global para Compromisos.

- Margen Global para Pagos.

- Examen/revisión intermedia que concluyó en julio de 2017.

- Revisión técnica para ajustar las dotaciones de la política de cohesión en 2016.

Anualmente, la Comisión procede al ajuste técnico del marco financiero para el año siguiente con el fin de ajustarlo a la inflación y a los últimos datos disponibles de RNB, dado que el marco financiero plurianual se expresa en precios constantes de 2011. Los techos del gasto a precios corrientes se establecen utilizando el deflactor fijo del $2 \%$ anual, de acuerdo con lo previsto en el artículo 6 (2) del Reglamento del Marco Financiero Plurianual. Al mismo tiempo, la Comisión calcula el margen disponible dentro del techo de recursos propios, el importe absoluto del margen para imprevistos, el margen global para pagos y el margen global para compromisos. Además, el subtecho de la Rúbrica 2 relativo a los gastos relacionados con el mercado y los pagos directos se reajustan tras $D$ 
las transferencias entre el primer pilar y el desarrollo rural (el importe total del techo de la Rúbrica 2 no cambia).

El importe máximo de los recursos propios está actualmente fijado en el 1,20\% de la RNB (SEC 2010), y el techo de los créditos de compromisos, en el $1,26 \%$ de la RNB (SEC 2010).

El Reino Unido notificó su intención de retirarse de la Unión Europea el 30 de marzo de 2019 (que finalmente ha tenido lugar el 31 de enero de 2020). A efectos del ajuste técnico del MFP 2014-2020, el Reino Unido se ha contado como si fuera un Estado miembro, de conformidad con los acuerdos adoptados entre ambas partes. El punto 59 del informe conjunto de los negociadores de la Unión Europea y del Gobierno del Reino Unido (de 8 de diciembre de 2017) y la Comunicación de la Comisión al Consejo Europeo (COM (2017) 784 final), prevén que el Reino Unido contribuirá y participará en la ejecución de los presupuestos anuales de la Unión de los años 2019 y 2020 como si todavía formase parte de ella.

El marco financiero plurianual 2014-2020 se estructura en las siguientes rúbricas:

Rúbrica 1. Crecimiento inteligente e integrador. Se subdivide en dos componentes diferentes pero estrechamente ligados entre sí.

1a. Competitividad para el crecimiento y el empleo. Comprende el gasto dirigido a garantizar la competitividad y la innovación en el mercado único, fomentar la investigación y el desarrollo tecnológico y las TIC, impulsar el transporte, la energía y las redes europeas sostenibles, mejorar la calidad de la educación y la formación, unido al desarrollo de un programa de política social que ayude a la sociedad europea a anticipar y gestionar los cambios.

1b. Cohesión económica, social y territorial, diseñado para impulsar la convergencia de las regiones y los Estados miembros menos desarrollados, para complementar la estrategia europea de desarrollo sostenible fuera de las regiones menos prósperas y para apoyar la cooperación interregional.

Rúbrica 2. Crecimiento sostenible: recursos naturales. Incluye la política agraria y pesquera común y la de desarrollo rural, además de medidas relacionadas con el medioambiente.

Rúbrica 3. Seguridad y ciudadanía. Refleja la importancia creciente de determinadas áreas en las que la Unión está adquiriendo nuevas competencias: justicia, protección de fronteras, políticas de inmigración y asilo, salud pública y protección de los consumidores, cultura, juventud e información y diálogo con los ciudadanos.

Rúbrica 4. Una Europa global. Cubre todas las acciones exteriores, incluyendo los instrumentos de preadhesión y las actuales reservas para ayudas de emergencia y garantía de préstamos.

Rúbrica 5. Administración. Comprende el gasto administrativo de todas las instituciones. En esta rúbrica también se recogen los gastos de pensiones y los gastos de las escuelas europeas.

Rúbrica 6. Compensaciones. Incluye cantidades destinadas a compensar los efectos relacionados con la adhesión de Croacia.

En el Cuadro 1 se presenta el marco financiero plurianual 2014-2020, a precios corrientes, para la UE-28 resultante del ajuste técnico para 2019. 
CUADRO 1

MARCO FINANCIERO PLURIANUAL 2014-2020 AJUSTADO PARA EL EJERCICIO 2019*

(Millones de euros corrientes)

\begin{tabular}{|c|c|c|c|c|c|c|c|c|}
\hline Créditos de compromiso & 2014 & 2015 & 2016 & 2017 & 2018 & 2019 & 2020 & Total \\
\hline 1. Crecimiento inteligente e integrador & 52.756 & 77.986 & 69.304 & 73.512 & 76.420 & 79.924 & 83.661 & 513.563 \\
\hline 1.a. Competitividad para el crecimiento y el empleo & 16.560 & 17.666 & 18.467 & 19.925 & 21.239 & 23.082 & 25.191 & 142.130 \\
\hline 1.b. Cohesión económica, social y territorial & 36.196 & 60.320 & 50.837 & 53.587 & 55.181 & 56.842 & 58.470 & 371.433 \\
\hline 2. Crecimiento sostenible: recursos naturales & 49.857 & 64.692 & 64.262 & 60.191 & 60.267 & 60.344 & 60.421 & 420.034 \\
\hline Del cual: gastos de mercado y pagos directos & 43.779 & 44.190 & 43.951 & 44.146 & 44.163 & 43.881 & 43.888 & 307.998 \\
\hline 3. Seguridad y ciudadanía & 1.737 & 2.456 & 2.546 & 2.578 & 2.656 & 2.801 & 2.951 & 17.725 \\
\hline 4. Europa global & 8.335 & 8.749 & 9.143 & 9.432 & 9.825 & 10.268 & 10.510 & 66.262 \\
\hline 5. Aministración & 8.721 & 9.076 & 9.483 & 9.918 & 10.346 & 10.786 & 11.254 & 69.584 \\
\hline Del cual: gasto administrativo de las instituciones & 7.056 & 7.351 & 7.679 & 8.007 & 8.360 & 8.700 & 9.071 & 56.224 \\
\hline 6. Compensaciones & 29 & 0 & 0 & 0 & 0 & 0 & 0 & 29 \\
\hline Total créditos de compromiso & 121.435 & 162.959 & 154.738 & 155.631 & 159.514 & 164.123 & 168.797 & 1.087.197 \\
\hline En porcentaje de RNB & 0,90 & 1,17 & 1,05 & 1,04 & 1,02 & 1,00 & 0,99 & 1,02 \\
\hline Total créditos de pago & 135.762 & 140.719 & 130.694 & 126.492 & 154.565 & 166.709 & 172.201 & 1.027 .142 \\
\hline En porcentaje de RNB & 1,01 & 1,01 & 0,88 & 0,84 & 0,98 & 1,01 & 1,01 & 0,96 \\
\hline Margen disponible (\%) & 0,22 & 0,22 & 0,35 & 0,39 & 0,22 & 0,19 & 0,19 & 0,26 \\
\hline Límite máx. RRPP en porcentaje de RNB & 1,23 & 1,23 & 1,23 & 1,23 & 1,20 & 1,20 & 1,20 & 1,22 \\
\hline
\end{tabular}

\section{El Presupuesto General de la Unión Europea para 2019}

El presupuesto de 2019 se aprobó definitivamente el 12 de diciembre de 2018, por acuerdo de las tres autoridades presupuestarias, tras el diálogo tripartito, del 4 de diciembre de 2018, entre el Parlamento Europeo y el Consejo, en el que alcanzaron un acuerdo ad referendum sobre un proyecto de conclusiones conjuntas.

El presupuesto inicialmente aprobado para 2019 ascendió a 165.795,6 millones de euros en créditos de compromiso, lo que representa el $1,01 \%$ de la RNB de la UE, y supone un aumento del 3,2\% con respecto al presupuesto final de 2018. Los créditos de pago se situaron en 148.198,9 millones de euros, importe que representa el $0,90 \%$ de la RNB de la UE y que supone un aumento del $2,4 \%$ en relación con el presupuesto final de 2018.
En estas cifras están incluidos los importes destinados a la Reserva de Ayuda de Emergencia, al Fondo Europeo de Adaptación a la Globalización y al Fondo de Solidaridad de la UE, que no se incluyen en el marco financiero plurianual. Deduciendo los importes señalados fuera del marco, las cuantías aprobadas dejan disponible, respecto de los límites previstos para 2019 en el MFP 2014-2020, un margen de 1.291,1 millones de euros.

El presupuesto inicialmente aprobado ha sufrido modificaciones a lo largo del ejercicio 2019, mediante la aprobación de tres presupuestos rectificativos (PR), que en algunos casos se han referido a revisiones de las previsiones de ingresos $y$, en otros, a modificaciones de los gastos del presupuesto de la UE.

EI PR 1/2019 ha tenido por objetivo consignar en el presupuesto 2019 el excedente del ejercicio 2018, por un importe de 1.803 millones de euros, lo que produjo una disminución en $\triangleright$ 
la contribución total de los 28 Estados miembros a la financiación del Presupuesto UE 2019.

EI PR 2/2019 refuerza con 100 millones de euros adicionales en créditos de compromiso las líneas Horizonte 2020 y Erasmus+, financiándose con cargo al margen global para compromisos, dada la falta de margen de la Rúbrica 1a.

El PR 3/2019 tiene como objetivo prestar asistencia a Rumanía, Italia y Austria por las catástrofes naturales sufridas en estos Estados miembros en 2018, movilizando para ello el Fondo de Solidaridad de la UE por un importe de 293,55 millones de euros tanto en créditos de compromiso como en créditos de pago.

La financiación del Presupuesto General de la Unión Europea para 2019 se ha realizado de acuerdo con la Decisión del Consejo, de 26 de mayo de 2014, sobre el sistema de recursos propios de la Unión Europea (2014/335/UE, Euratom).

De acuerdo con esta decisión, los recursos propios de que dispone la Unión Europea para financiar sus políticas están constituidos por:
1. Los recursos propios tradicionales (derechos de aduana). Los Estados miembros retienen el $20 \%$ de esas cantidades en concepto de gastos de recaudación.

2. El recurso IVA, mediante la aplicación de un tipo uniforme del $0,30 \%$ a la base imponible del IVA armonizada, que no podrá exceder del $50 \%$ de la renta nacional bruta para cada Estado miembro. El tipo máximo de referencia para Alemania, Países Bajos y Suecia se fija en el 0,15 para el periodo 2014-2020.

3. El recurso RNB se realiza mediante la aplicación de un tipo uniforme, que se determinará en función del total de todos los demás ingresos, a la RNB de cada Estado miembro.

La aportación por este recurso se ve incrementada por las compensaciones reconocidas a favor de determinados Estados miembros (que España contribuye a financiar), que se formalizan como segundos tramos de la aportación por recurso RNB:

\begin{tabular}{|c|c|c|c|c|c|c|}
\hline \multicolumn{7}{|c|}{$\begin{array}{c}\text { CUADRO } 2 \\
\text { PRESUPUESTO GENERAL DE LA UNIÓN EUROPEA PARA } 2019 \\
\text { (Millones de euros) }\end{array}$} \\
\hline \multirow[t]{2}{*}{ Categorías de gasto } & \multicolumn{2}{|c|}{$\begin{array}{c}\text { Pto. } 2018+\text { Pr. } \\
1 \text { a } 6 / 2018\end{array}$} & \multicolumn{2}{|c|}{$\begin{array}{c}\text { Pto. } 2019+\text { Pr. } \\
1 \text { a } 3 / 2019\end{array}$} & \multicolumn{2}{|c|}{$\begin{array}{c}\text { Variación en \% } \\
2019 / 2018\end{array}$} \\
\hline & C.C. & C.P. & C.C. & C.P. & C.C. & C.P. \\
\hline 1. Crecimiento inteligente e integrador & $77.531,8$ & $66.622,6$ & $80.627,4$ & $67.556,9$ & 3,99 & 1,40 \\
\hline 1a. Competitividad para el crecimiento y el empleo & $21.999,6$ & $20.095,3$ & $23.435,4$ & $20.521,5$ & 6,53 & 2,12 \\
\hline 1b. Cohesión económica, social y territorial & $55.532,2$ & $46.527,3$ & $57.192,0$ & $47.035,4$ & 2,99 & 1,09 \\
\hline 2. Crecimiento sostenible: recursos naturales & $59.238,6$ & $56.041,0$ & $59.642,1$ & $57.399,9$ & 0,68 & 2,42 \\
\hline Del cual: gastos de mercado y pagos directos & $43.233,1$ & $42.561,1$ & $43.191,9$ & $43.116,4$ & $-0,10$ & 1,30 \\
\hline 3. Seguridad y ciudadanía & $3.493,2$ & $2.980,7$ & $3.786,6$ & $3.527,4$ & 8,40 & 18,34 \\
\hline 4. Europa global & $10.068,8$ & $8.906,1$ & $11.319,3$ & $9.358,3$ & 12,42 & 5,08 \\
\hline 5. Administración & $9.665,5$ & $9.666,3$ & $9.943,0$ & $9.944,9$ & 2,87 & 2,88 \\
\hline Total MFP & $159.997,9$ & $144.216,7$ & $165.318,3$ & $147.787,4$ & 3,33 & 2,48 \\
\hline Otros instrumentos especiales fuera del MFP $\left({ }^{*}\right)$ & 698,5 & 551,2 & 870,8 & 705,1 & 24,66 & 27,90 \\
\hline Total dentro y fuera del MFP & $160.696,5$ & $144.767,9$ & $166.189,1$ & $148.492,4$ & 3,42 & 2,57 \\
\hline
\end{tabular}


- La compensación británica, reconocida al Reino Unido desde 1984, y que supone una reducción en sus aportaciones en un importe aproximado a las dos terceras partes de su saldo negativo. El importe que el Reino Unido deja de ingresar es soportado por el resto de Estados miembros en clave RNB, con la excepción de Alemania, Austria, Suecia y Países Bajos, que soportan solo la cuarta parte de su cuota; lo que ahorran estos cuatro países es financiado por los 23 Estados restantes.

- Las reducciones brutas anuales en las contribuciones por recurso RNB para el periodo 2014-2020 de Dinamarca, 130 millones de euros; Países Bajos, 695 millones; y Suecia, 185 millones; además, Austria cuenta con reducciones de 30 millones en 2014 , 20 millones en 2015 y 10 millones en 2016. Todo ello a precios de 2011. El importe conjunto de estas reducciones es soportado por todos los Estados miembros (incluidos Dinamarca, Suecia, Países Bajos y Austria, que participan así en la financiación de su propia compensación) en clave RNB.

Aparte de los recursos propios, existen otros ingresos que proceden, entre otros, de los impuestos pagados por los funcionarios, de las multas impuestas por la Comunidad a empresas y de los intereses de demora.

El presupuesto de la Unión debe estar equilibrado, es decir, debe contar con ingresos suficientes para financiar todos los gastos ya que la Unión no está autorizada a recurrir al endeudamiento para financiarlos.

CUADRO 3

FINANCIACIÓN DEL PRESUPUESTO GENERAL DE LA UNIÓN EUROPEA (Millones de euros)

\begin{tabular}{|c|c|c|c|c|c|}
\hline \multirow{2}{*}{ Títulos y capítulos } & \multicolumn{2}{|c|}{2018} & \multirow{2}{*}{$\begin{array}{l}2019 \\
\text { Pto. }^{2}\end{array}$} & \multirow{2}{*}{$\begin{array}{l}2019 / \text { Pto. } \\
2018\end{array}$} & \multirow{2}{*}{$\begin{array}{l}\text { 2019/Ejec. } \\
2018\end{array}$} \\
\hline & Pto. ${ }^{1}$ & Ejecución & & & \\
\hline 1. Recursos propios & 142.364 & 142.330 & 144.795 & $1,7 \%$ & $1,7 \%$ \\
\hline 1.1. Cotizaciones azúcar e isoglucosa & 93 & 85 & $\mathrm{pm}$ & - & - \\
\hline 1.2. Derechos aduana y otros & 20.165 & 20.317 & 21.471 & $6,5 \%$ & $5,7 \%$ \\
\hline 1.3. Recurso IVA & 17.149 & 17.133 & 17.739 & $3,4 \%$ & $3,5 \%$ \\
\hline 1.4. Recurso RNB & 105.143 & 104.979 & 105.585 & $0,4 \%$ & $0,6 \%$ \\
\hline 1.5. Compensación Reino Unido & 0 & 19 & 0 & - & - \\
\hline 1.6. Reducción bruta recurso RNB & 0 & 6 & 0 & - & - \\
\hline 3. Excedentes, saldos y ajustes & 556 & 581 & 1.803 & - & - \\
\hline 4. Ingresos relativos al personal & 1.547 & 1.542 & 1.607 & $3,8 \%$ & $4,2 \%$ \\
\hline 5. Ingresos del funcionamiento admvo de las instituciones & 45 & 563 & 25 & $-44,3 \%$ & $-95,6 \%$ \\
\hline 6. Contribuciones relativas a acuerdos y programas de la UE & 110 & 12.777 & 130 & $18,2 \%$ & $-99,0 \%$ \\
\hline 7. Intereses de demora y multa & 115 & 1.473 & 115 & $0,0 \%$ & $-92,2 \%$ \\
\hline 8. Empréstitos y préstamos & 6 & 39 & 3 & $-52,9 \%$ & $-92,8 \%$ \\
\hline 9. Ingresos diversos & 25 & 13 & 15 & $-40,0 \%$ & $15,4 \%$ \\
\hline Total ingresos & 144.768 & 159.318 & 148.492 & $2,6 \%$ & $-6,8 \%$ \\
\hline \multirow{2}{*}{\multicolumn{6}{|c|}{$\begin{array}{l}{ }_{1}^{1} \text { Presupuesto } 2018 \text {, incluidos los presupuestos rectificativos (PR) n. } 1 / 2018 \text { a 6/2018. } \\
{ }^{2} \text { Presupuesto 2019, incluidos los presupuestos rectificativos (PR) n. } 1 / 2019 \text { a 3/2019. } \\
\text { pm: pro memoria }\end{array}$}} \\
\hline & & & & & \\
\hline
\end{tabular}




\section{Saldo financiero España-Unión Europea en el año 2019}

Los flujos monetarios de ingresos procedentes de la Unión Europea y de pagos efectivamente realizados por España en el año 2019 arrojan un saldo financiero negativo que asciende a $-1.376,2$ millones de euros, frente a $-235,8$ millones en 2018 , como puede verse en el Cuadro 4.

\section{CUADRO 4}

SALDO FINANCIERO ESPAÑA-UNIÓN EUROPEA (Millones de euros)

\begin{tabular}{|c|c|c|}
\hline & 2018 & 2019 \\
\hline $\begin{array}{l}\text { Aportación española a la } \\
\text { Unión Europea }\left(^{*}\right)\end{array}$ & $11.682,4$ & $12.215,6$ \\
\hline $\begin{array}{l}\text { Ingresos procedentes de la } \\
\text { Unión Europea }\left(^{*}\right)\end{array}$ & $11.446,6$ & $10.839,4$ \\
\hline Saldo financiero & $-235,8$ & $-1.376,2$ \\
\hline \multicolumn{3}{|c|}{$\begin{array}{l}\text { (*) Aportación neta tras descontar los gastos de percepción de los } \\
\text { recursos propios tradicionales. Los ingresos tampoco incluyen este } \\
\text { importe. }\end{array}$} \\
\hline \multicolumn{3}{|c|}{ Fuente: DG del Tesoro y DG de Fondos Europeos. } \\
\hline
\end{tabular}

La aportación española a la Unión Europea en el año 2019 aumenta un 4,56\% respecto a 2018 y los ingresos procedentes de aquellas han disminuido un $5,30 \%$. El saldo financiero resultante es de signo negativo para España, empeorando respecto a 2018 en 1.140,4 millones de euros. Los componentes del saldo financiero en el periodo 2009-2019 pueden verse en el Cuadro 6 (en página siguiente).

\section{Aportación española a la Unión Europea en el año 2019}

En el Cuadro 5 se recoge la aportación española al PGUE y al Fondo Europeo de Desarrollo (FED) durante el año 2019.

Los pagos realizados por España a la Unión Europea en el año 2019, en términos de caja,
CUADRO 5

APORTACIÓN ESPAÑOLA A LA UNIÓN EUROPEA (Millones de euros)

\begin{tabular}{|l|r|r|}
\hline \multicolumn{1}{|c|}{ Conceptos } & \multicolumn{1}{c|}{$\mathbf{2 0 1 8}$} & \multicolumn{1}{c|}{$\mathbf{2 0 1 9}$} \\
\hline Recursos propios tradicionales netos & $1.535,8$ & $1.585,4$ \\
Recurso IVA neto & $1.691,7$ & $1.598,7$ \\
Recurso RNB neto & $8.050,8$ & $8.622,4$ \\
FED & 356,9 & 372,7 \\
Aportación por prestación de servicios & 1,4 & 0,7 \\
Intereses de demora & 0,0 & 1,5 \\
Otros pagos a la UE & 45,8 & 34,1 \\
\hline Total & $\mathbf{1 1 . 6 8 2 , 4}$ & $\mathbf{1 2 . 2 1 5 , 6}$ \\
\hline Fuente: DG del Tesoro y DG de Fondos Europeos. \\
\hline \multicolumn{2}{|l|}{} \\
\hline
\end{tabular}

ascendieron a 12.215,6 millones de euros, frente a $11.682,4$ millones en 2018 , lo que representa un aumento del $4,56 \%$; estos pagos tienen su reflejo en los capítulos 4 (transferencias corrientes) y 7 (transferencias de capital: FED) de la sección 34 del presupuesto de gastos del Estado español, a excepción del pago de intereses de demora que transitan por el capítulo 3 de gastos financieros de dicha sección. Los distintos componentes de estos pagos y su evolución en el año 2019 han sido los siguientes.

Los recursos propios tradicionales han crecido un $3,23 \%$ respecto a 2018 , debido, principalmente, al aumento en la recaudación de los derechos de aduana del $3,35 \%$.

La aportación por recurso IVA neto disminuye un $5,50 \%$ en relación con el año 2018; en 2019 el tipo aplicable a la base IVA ha sido del $0,30 \%$.

Cabe señalar que, en el Comité de Conciliación entre el Parlamento Europeo y el Consejo celebrado en noviembre de 2019, no se logró alcanzar un acuerdo sobre el presupuesto rectificativo 4/2019 (ni sobre la segunda versión presentada por la Comisión PR 5/2019), que recogían, por el lado de los ingresos, la revisión de las bases IVA y RNB, los importes a obtener de recursos propios tradicionales y el cheque británico; y, por el lado del gasto, $D$ 


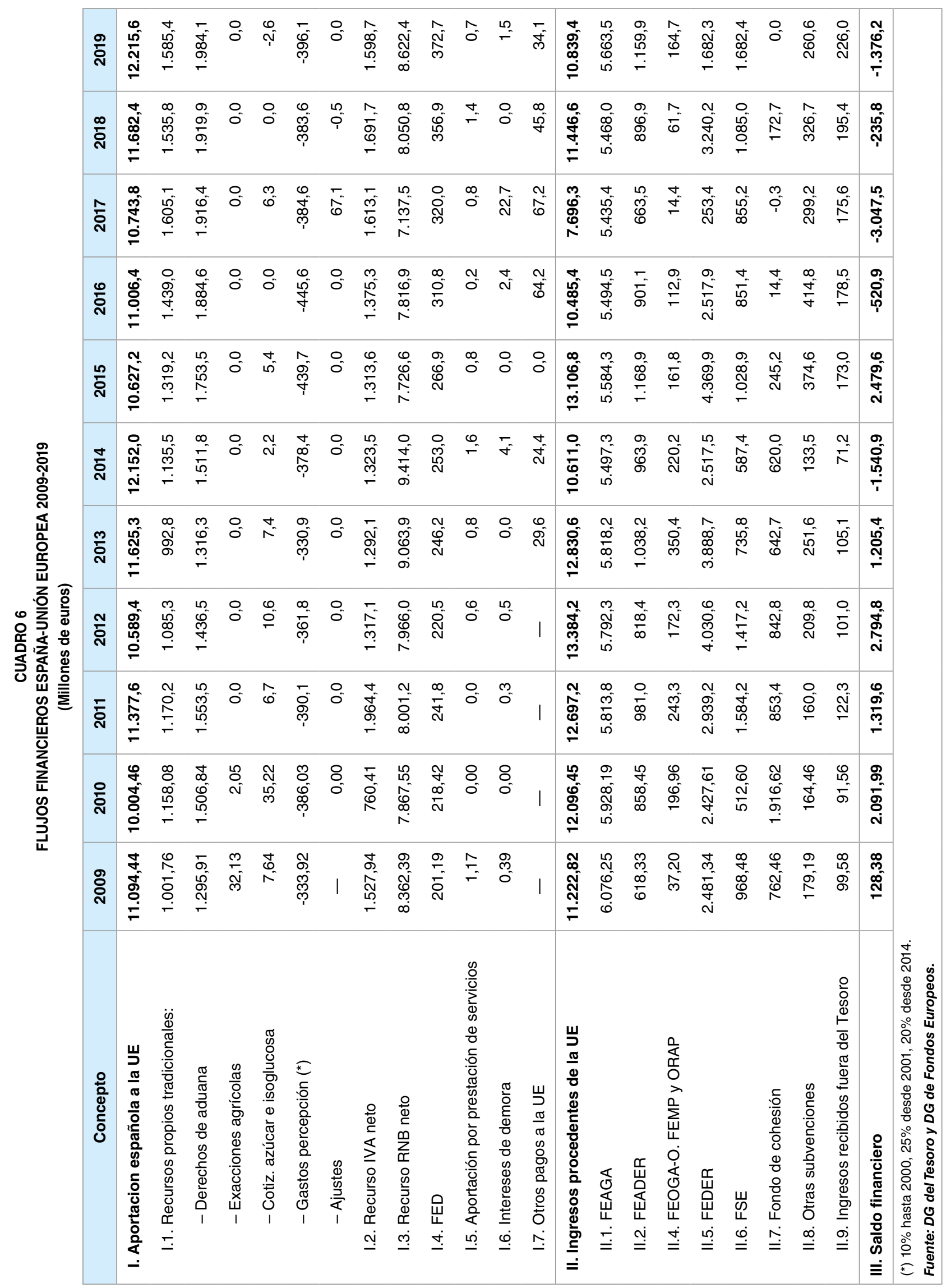


una reducción en el volumen de créditos de pago a financiar en 2019.

La actualización de las bases IVA recogida en dicho rectificativo habría supuesto un incremento en la aportación española en 2019 por IVA de 109,7 millones de euros.

Por otra parte, de acuerdo con el artículo 10 ter del Reglamento 2016/804, la Comisión, antes del 1 de febrero, informa a los Estados miembros de sus saldos IVA y RNB, calculados conforme a lo previsto en el Reglamento (CEE, Euratom) 1553/89 y el Reglamento (CE, Euratom) 1287/2003; el importe total de estos saldos se distribuye entre los Estados miembros de acuerdo con la participación de cada uno en el total RNB de la UE; finalmente, el importe que corresponde a cada Estado en esta redistribución se deduce del importe de su saldo total IVA y RNB, lo que da como resultado el importe neto de saldo a pagar o reintegrar, por cada Estado miembro, el primer día hábil del mes de junio.

En 2019 la suma total de los saldos derivados de la actualización de las bases IVA y RNB de todos los Estados miembros ha sido positiva, por un importe de 3.442,30 millones de euros, es decir, a reembolsar a los Estados miembros. De acuerdo con nuestra participación en la RNB de la UE, a España le ha correspondido en 2019 el 7,65\% de esa cuantía, es decir, 263,39 millones de euros (Cuadro 7).

El importe pagado por IVA a la UE en el año 2019 incluye la liquidación del saldo del ejercicio 2017, por el cálculo definitivo de la base armonizada de dicho año (-9,80 millones de euros), y el saldo de ejercicios anteriores de 2011 a 2016, por importe de 0,28 millones de euros, como consecuencia de los controles realizados por la Comisión Europea; el resultado final de ello ha sido una devolución a España por IVA, en el mes de junio, de 9,52 millones de euros.
Los pagos realizados por España en concepto de recurso RNB durante el año 2019 registraron un aumento del $7,10 \%$ respecto a los de 2018; conviene recordar que el importe global de este recurso al tipo uniforme, en el presupuesto de la UE, se calcula como la diferencia entre el total de gasto del presupuesto UE y el resto de los ingresos, ya que el presupuesto comunitario no puede presentar déficit, y el reparto de esa cantidad entre los Estados miembros se calcula de forma proporcional a sus respectivas RNB.

La aportación española en concepto de RNB a la UE a lo largo de 2019 comprende los pagos de la RNB al tipo uniforme, los pagos por la compensación británica, los pagos adicionales por la reducción bruta en la aportación RNB de Dinamarca, Países Bajos y Suecia, y, en el mes de diciembre, la aportación adicional por RNB para financiar la no participación de algunos Estados miembros en determinadas políticas.

Además, al igual que en el IVA, el importe pagado por RNB a la UE en el año 2019 incluye el pago del saldo RNB del ejercicio 2017 por el cálculo definitivo de la RNB de dicho año (36,96 millones de euros), y del saldo de ejercicios anteriores de 2010 a 2016, por importe de 16,45 millones de euros, como consecuencia de las revisiones efectuadas por el INE; también incluye el importe de la devolución efectuada por la Comisión a España en el mes de junio, tras la redistribución del saldo total IVA+RNB de toda la UE, que en el caso de España ha ascendido a -263,39 millones de euros.

Otro componente de los pagos realizados por España en el año 2019 es la contribución al FED, que ha experimentado un aumento del 4,44\% respecto a 2018. En 2019 los pagos al FED se han realizado con cargo al 10. y 11.. ${ }^{\circ}$ FED. $\quad \square$ 


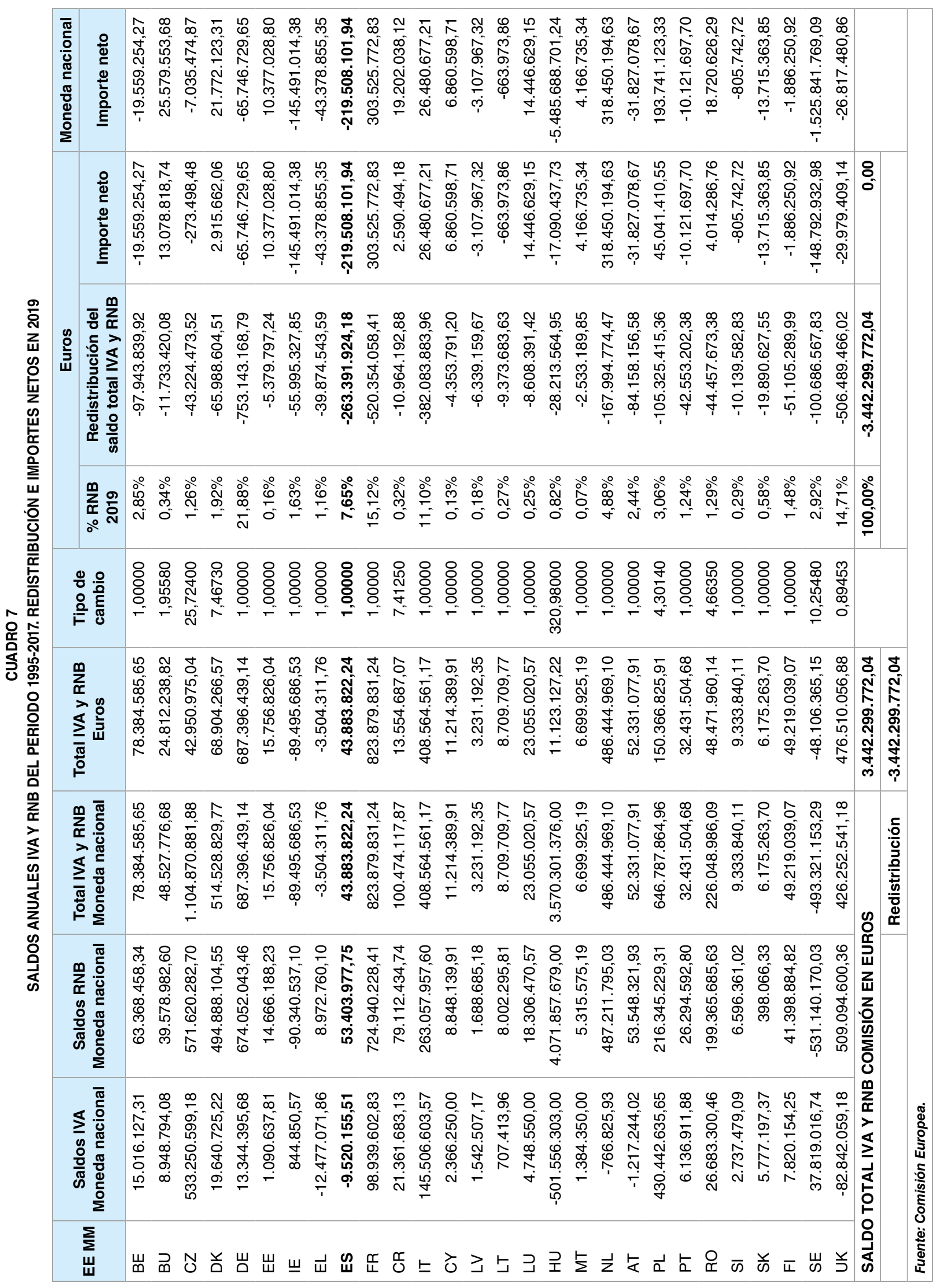


El Fondo Europeo de Desarrollo no está integrado en el PGUE y se financia con las contribuciones de los Estados miembros de acuerdo a una clave de financiación aprobada para cada fondo, clave que se va aproximando cada vez más a la clave RNB. A España le corresponde financiar el $7,85 \%$ del décimo FED y el $7,93 \%$ del undécimo FED.

El importe global para el undécimo FED que cubre el periodo 2014-2020 se fijó en el Consejo Europeo de febrero de 2013, junto con el Marco Financiero Plurianual de la UE.

Se realizan tres contribuciones anuales (enero, julio y noviembre), fijadas mediante Decisión del Consejo, publicada en el Diario Oficial de la UE, debiendo hacerse efectivas en los veintiún días naturales siguientes a su adopción.

El resto de pagos al Presupuesto de la UE en 2019 han sido los siguientes:

- La aportación para financiar nuestra participación en los gastos del servicio de interpretación para sufragar parcialmente el coste derivado de la participación de los intérpretes en diversas reuniones del Consejo, que se ha situado en 0,67 millones de euros.

- El importe pagado en 2019 por España para el Fondo de Refugiados para Turquía asciende a 23,78 millones de euros.

- En el concepto que recoge los «Intereses de demora a la UE» se ha pagado un importe de 1,50 millones de euros.

- Adicionalmente, y fuera de la sección 34 del presupuesto del Estado, se abonó en 2019 un importe de 10,36 millones de euros, correspondiente al pago de la primera multa coercitiva semestral impuesta por la Sentencia del Tribunal de Justicia de la Unión Europea en el asunto
C-205/17 Comisión/ Reino de España (Tratamiento de aguas residuales urbanas).

\section{Ingresos procedentes de la Unión Europea en el año 2019}

El importe total de fondos recibidos por España de la Unión Europea durante el año 2019 ha ascendido a 10.839,4 millones de euros, lo que representa un descenso respecto al año 2018 del 5,30\% (Cuadro 8).

\begin{tabular}{|c|c|c|}
\hline \multicolumn{3}{|c|}{$\begin{array}{l}\text { CUADRO } 8 \\
\text { INGRESOS PROCEDENTES DE LA UNIÓN EUROPEA } \\
\text { (Millones de euros) }\end{array}$} \\
\hline Conceptos & 2018 & 2019 \\
\hline FEAGA & $5.468,00$ & $5.663,50$ \\
\hline FEADER & 896,90 & $1.159,90$ \\
\hline FEMP y ORAP & 61,70 & 164,70 \\
\hline FEDER & $3.240,20$ & $1.682,30$ \\
\hline FSE & $1.085,00$ & $1.682,40$ \\
\hline Fondo de Cohesión & 172,70 & 0,00 \\
\hline Otras subvenciones & 326,70 & 260,60 \\
\hline Ingresos fuera de Tesoro & 195,40 & 226,00 \\
\hline Total & $11.446,60$ & $10.839,40$ \\
\hline
\end{tabular}

En este periodo 2014-2020, se mantiene la estructura en dos pilares de la PAC, el primero referido a la política de pagos directos, de precios y de mercados, que se financiará con el Fondo Europeo Agrícola y de Garantía (FEAGA) y en exclusiva a través del presupuesto de la UE; mientras que el segundo, referido a la política de desarrollo rural, se instrumenta con el Fondo Europeo Agrícola de Desarrollo Rural (FEADER) y es cofinanciado por los Estados miembros.

En materia de las políticas marítima y pesquera, en el nuevo periodo se crea el Fondo Europeo Marítimo y de Pesca (FEMP), destinado a ayudar a los pescadores en la transición a la pesca sostenible y a subvencionar $\triangleright$ 
a las comunidades costeras en la diversificación de sus economías. Este fondo sustituye al Fondo Europeo de Pesca (FEP) del periodo anterior.

EI FEADER y el FEMP constituyen, junto con el FEDER, el FSE y el Fondo de Cohesión, los denominados Fondos Estructurales y de Inversión Europeos (FEIE/EIE) y se inscriben en el mismo marco estratégico común establecido en el Reglamento 1303/2013.

Las transferencias del FEAGA a España, en el año 2019, ascienden a 5.663,5 millones de euros. En cuanto a las transferencias del FEADER, se sitúan en 1.159,9 millones de euros. Con ello, el total de fondos recibidos por España del FEADER y del FEAGA en 2019 asciende $6.823,4$ millones de euros, un $7,20 \%$ superior a lo recibido en 2018.

Por lo que se refiere a la política de pesca y otros recursos agrarios y pesqueros (sanidad animal y otros), España ha ingresado en 2019 transferencias por importe de 164,7 millones de euros, un $167 \%$ más que en 2018.

El importe de las transferencias procedentes de los Fondos Estructurales (FEDER y Fondo Social Europeo) en 2019 se eleva a 3.364,7 millones de euros, lo que supone una reducción del 22,21 \% respecto de la cifra de 2018, que viene afectada por el ingreso en 2018 de los últimos saldos de cierre de los programas del periodo 2007-2013.

Por lo que se refiere al Fondo Europeo de Desarrollo Regional, los ingresos caen en 2019 un $48,08 \%$ respecto a 2018 . $Y$ los ingresos recibidos por el Fondo Social Europeo crecen en 2019 un 55,06 \% respecto a 2018.

España no recibe, en el periodo 2014-2020, ayudas del Fondo de Cohesión. No obstante, en los primeros años del actual periodo, España ha estado recibiendo ingresos procedentes de la ayuda transitoria del régimen de phasing out del Fondo de Cohesión en el Objetivo de Convergencia al que tuvo acceso en el periodo 2007-2013, junto con pequeños saldos correspondientes al cierre de proyectos pertenecientes al periodo 2000-2006. En 2018 España ingresó 172,7 millones de euros de las últimas liquidaciones de proyectos beneficiarios del Fondo de Cohesión, sin que queden pendientes importes a partir de 2019.

Además de las transferencias para la financiación de las grandes políticas agraria y de cohesión, el presupuesto europeo prevé toda una lista de programas e instrumentos que ofrecen cobertura a los diferentes Estados miembros para financiar una serie de actuaciones enmarcadas en las políticas de educación, inmigración, investigación y desarrollo, etcétera. El importe recibido por estos conceptos en 2019 se sitúa en 486,6 millones de euros. No obstante, estas cifras no incluyen una buena parte de las transferencias en materia de Investigación y Desarrollo, con las que la UE contribuye a financiar entidades públicas y privadas y que se reciben directamente en estas entidades. 